\title{
Le Veilleur
}

\author{
Peggy KamuF \\ University of Southern California \\ escrituraeimagen@filos.ucm.es
}

\section{Resumen}

Entre dos lenguas, la traducción de veille o veiller por wake o to wake produce una convergencia inesperada entre la vigilia (veille) y la estela (sillage). A partir de ella la autora traza en la obra de Derrida una escritura del wake que teje en ella otro modo de vigilancia crítica como escucha atenta a la interrupción, al cambio de tono y a la irrupción del otro. Esa vigilancia no se queda en la hiper-crítica ilustrada sino que, más allá de la oposición metafísica entre el sueño y la vigilia, vigila el sueño para velar por el porvenir.

Palabras clave: veille, sillage, vigilancia, escritura del wake, crítica, interrupción, sueño.

\begin{abstract}
Between two languages, the translation of veille or veiller as wake or to wake creates an unexpected convergence between wake (veille) and wake (sillage). Based on it, the author traces in Derrida's work a writing of the wake which weaves in it another form of critical vigilance as attentive listening to interruption, change in tone and the irruption of the other. This watchfulness does not end in the enlightened hyper critique, but, beyond the metaphysical opposition between dream and wake, it looks after the dream to take care of what is "to-come".
\end{abstract}

Key words: Veille, sillage, watchfulness, writing of the wake, critique, interruption, dream. 
... pour veiller à l'avenir, il faudrait tout recommencer.

Spectres de Marx

Pardonnez-moi de commencer par une espèce de récit. Il servira de bouche-trou dans cette intervention que j'ai découpée dans un texte plus long, trop long pour notre colloque.

Il s'agit de l'oubli d'un mot, ou plutôt d'une homophonie rassemblant au moins deux mots en anglais. L'un d'eux serait une traduction possible du substantif veille ou veillée et le verbe veiller en français: wake et to wake. Cependant, l'autre wake n'a rien à voir avec toute cette famille. Son meilleur équivalent en français serait le sillage. Mon récit concerne une répétition de ce jeu homophonique à trente ans d'intervalle. Quand, assez récemment, je m'apprêtais à recueillir divers écrits que j'avais consacrés depuis dix ans à la pensée, l'enseignement et l'amitié de Jacques Derrida, une partie du titre pour ce recueil s'était d'avance fixée dans mon esprit sans que je puisse dire qu'il fut choisi après réflexion. Plutôt, la phrase « the wake of Jacques Derrida » m'a intimé de la recopier, comme sous l'effet d'une dictée. Sans doute, l'expérience d'écrire sous dictée est assez banale; ce n'est donc pas cette répétition qu'il me fallait commencer par raconter ici. Mais j'avais oublié qu'à peu près trente ans plus tôt, ce fut la même homophonie sous dictée qui m'avait donné un titre pour une première tentative d'écrire sur le travail de Derrida: ce texte, «Abraham's Wake », traitait de « Fors», la préface que Derrida avait faite pour Le Verbier de l'Homme aux Loups, de Nicolas Abraham et Maria Torok. Quand cet oubli d'un titre fut réparé, je fus saisie par l'apparence que ce nom anglais de « wake » avait surveillé depuis plus de trente ans une expérience d'écrire dans les parages de l'oeuvre de Jacques Derrida. Cette expérience a été, elle aura été «a wake ». Je m'interrogeais donc: qu'est-ce qu'un wake, que veut dire to wake?

Afin de gagner du temps et puisque cela ne peut passer sans dommages par la traduction, je laisserai tomber à peu près tout ce qu'un dictionnaire de la langue anglaise enseigne sur ces mots. Au sens de veille, veillée, les usages de wake ressemblent plus ou moins à ceux qu'on connaît en français. Au sens de sillage aussi, mais évidemment seule la langue anglaise permet le croisement possible des homophones. A retenir de cette consultation lexicologique serait pourtant un glissement entre les rapports dans le temps -avant ou après- et qui peut s'exprimer aussi bien par des rapports dans l'espace -devant ou derrière- grâce au croisement avec la notion de sillage. Ainsi, wake, avec toutes ses possibilités entre temporisation et espacement, laisse penser à un site particulièrement propice quant à ses surgissements dans la langue pour ce que Derrida a nommé la différance. Par leur jeu s'installe un suspens du sens toujours possible par lequel ce qui vient après peut toujours précéder, et la trace d'un passage peut veiller à ce qui vient. Laisser un sillage der- 
rière et veiller à l'à-venir: ces « wakes » tirent dans des sens opposés et pourtant l'écriture est toujours capable de forger leur convergence et inscrire l'un dans ou à partir de l'autre.

Un passage de Moby Dick nous donnera une idée de cette convergence de l'écriture du wake. Je traduis :

tel le pilote qui se tient auprès de sa boussole, et note le cap précis alors visible, afin de toucher au point voulu la terre distante et invisible qu'on visitera plus tard, ainsi fait le pêcheur, à sa boussole, avec la baleine ; car, après avoir été poursuivie, et marquée avec diligence pendant plusieurs heures du jour, ensuite, quand la nuit cache le poisson, le sillage futur de la créature à travers l'obscurité est presqu'aussi fixé dans l'esprit avisé du chasseur, que la côte l'est pour le pilote. Ainsi, à l'adresse merveilleuse de ce chasseur, l'évanescence proverbiale d'une chose écrite dans l'eau [writ in water], un sillage, est compte tenu des fins désirées tout aussi fiable que la terre fixe. ${ }^{1}$

Écrivant qu'un sillage est (comme) l'écriture, Melville laisse lire aussi que l'écriture est (comme) un sillage tracé à travers le médium invisible de la pensée, se poursuivant "à travers l'obscurité » tel le chasseur qui veille à traquer la baleine. Ce qui converge ici, c'est le sillage et la veille, le wake avec la nuit et donc avec cet autre wake qui veille afin d'anticiper le « future wake ». Celui-ci -mais lequel ?est écrit d'avance, avant ou devant " l'évanescence proverbiale d'une chose écrite dans l'eau ». Melville signale une allusion à la phrase célèbre que le poète John Keats avait dictée pour son propre épitaphe : «Here lies one whose name was writ in water »; Repose ici quelqu'un dont le nom fut écrit dans l'eau. Les deux faces de l'homophonie, le sillage et la veillée des morts, l'écriture du wake les trace l'une dans ou sur l'autre.

Un mot encore et je termine ce récit. Ce mot revient depuis «Fors», texte auquel je fus renvoyée par mon oubli. Dès ses premières lignes, «Fors » demande ou se demande ce qu'il en est du lieu de l'écriture. Où a-t-elle lieu ? Sur quelle surface ? Sur quelle division entre le dehors et le dedans ? Ce devenir-insituable de l'écriture se profile entre deux questions, l'une dans le sillage de l'autre. Je lis cette ouverture de « Fors » :

Qu'est-ce qu'une crypte?

Et si j'écrivais ici sur elle ?2

\footnotetext{
${ }^{1}$ Herman Melville, Moby-Dick, or the Whale (London and New York: Norton, 1967), 453.

2 Derrida, "Fors : Les Mots anglés de Nicolas Abraham et Maria Torok, » in Abraham et Torok, Cryptonymie : Le Verbier de l'Homme aux Loups (Paris, Aubier-Flammarion, 1976), 9.
} 
Dans leur séquence minimale, ces questions s'avèrent étranges, uncanny même. Car «Qu'est-ce qu'une crypte ? », comme Derrida va le préciser, ne demande pas ce que c'est que "la crypte en général, mais celle-ci, toute singulière » (" Fors », 10). Ainsi, la question se détache de tout fonds de savoir commun ou général d'une langue dans laquelle le mot " crypte » serait ancré dans un concept. Au lieu d'un lieu fixe et connu, le lecteur se trouve isolé, comme sur une île déserte inconnue, le lieu d'une singularité, " celle-ci ». La deuxième question ne resserre les coordonnés de ce lieu qu'à les situer dans une conjonction avec l'écriture : Qu'est-ce qu'une crypte si je pouvais écrire sur elle ici, maintenant ? C'est le sens concret et non seulement abstrait d' " écrire sur » qu'il faudrait dès lors prendre en compte, c'està-dire, l'idée non seulement d'écrire sur un sujet ou à propos de quelque chose, par exemple des cryptes, mais aussi sur un support, une surface, la face externe d'une construction, par exemple, le mur d'une crypte, celle-ci. Si son support peut être une crypte, toujours singulière, alors qu'est ce qu'une écriture dont la condition de lisibilité pour l'autre est une telle situation singulièrement encryptée?

Ou bien, pour le dire autrement mais en des termes non moins cryptés, et pour moi d'abord :

Qu'est-ce qu'un wake?

Et si j'écrivais ici sur lui?

Fin du récit. Dans son sillage, je poursuis sur un autre ton.

$$
* * *
$$

Immanquablement, ou presque, Derrida assigne une valeur positive à l'état de vigilance; c'est le nom, si vous voulez, d'une des vertus les plus louées et prisées de son vocabulaire. ${ }^{3}$ Sans doute, cette évaluation ne lui est pas du tout unique car, dans une très grande mesure, c'est l'héritage de la philosophie moderne du moins depuis les Lumières. Surtout depuis Kant, la vigilance (de vigilo, vigilare: veiller, être éveillé) s'allie et se rend quasi synonyme avec la lumière de la raison critique qui chasse les ombres obscurantistes. C'était Kant, après tout, qui a fait le célèbre aveu que la lecture de « David Hume fut ce qui, il y a tant d'années, a interrompu mon sommeil dogmatique et dirigé mes enquêtes en philosophie spéculative dans

\footnotetext{
3 To take just a few examples: "He applies an extraordinary vigilance, a faultless erudition to the measure of the desire of the unconscious" (on Roger Laporte); "With a vigilance one could probably say operates at every instant ..." (on Emmanuel Lévinas); "For example, to analyze a genre or discursive code, or the rules of a particular social arrangement, and to do so with his meticulousness and vigilance ..." (on Roland Barthes) (Psyche I, 81, 166, 282); "A joy to rediscover, to discover in another way, the force and the exigency, the uncompromising vigilance, of a faithful thought" (on Philippe Lacoue-Labarthe), (Psyche II, 201); "while reading himself, while analyzing vigilantly his selftaste..." (on J.H. Miller) (“Justices," 715); "her friendly vigilance" (on Peggy Kamuf) (Alibi, xxv).
} 
un sens tout à fait différent ». ${ }^{4}$ Ce réveil de la philosophie critique, non-dogmatique n'a depuis cessé de résonner et nulle part de manière plus provocatrice pour la pensée que dans les écrits de Jacques Derrida.

Un héritage, comme Derrida a affirmé de façon la plus insistente par rapport à l'héritage de Marx et du marxisme, « est toujours la réaffirmation d'une dette mais une réaffirmation critique, sélective et filtrante » (Spectres, 150). Il paraît difficile d'étendre cette définition à la réaffirmation de la vigilance elle-même, qui est moins le legs d'un seul penseur que la condition ou la possibilité même de la réception et de l'héritage critiques. Néanmoins, l'acte principal de Spectres de Marx -la réaffirmation de l'héritage marxiste par la déconstruction- reste très pertinente et non seulement parce que Marx lui-même lègue une vigilance exemplaire devant tous les dogmatismes endormis de l'économie politique, de la théorie sociale ou de l'idéologie religieuse. Comme Derrida le rappelle, Marx aussi était forcément un héritier de cet « esprit des Lumières auquel il ne faut pas renoncer » (Spectres, 145). Mais l'hyper-vigilance du marxisme, cette «critique radicale, à savoir une démarche prête à son auto-critique » (ibid.), ne situe pas encore sa pertinence pour une évaluation de la réaffirmation critique et déconstructrice de cet héritage des Lumières chez Derrida. Cette pertinence s'attache plutôt à un autre « esprit du marxisme » auquel, Derrida avoue, « je ne serai jamais prêt à renoncer » :

Or s'il est un esprit du marxisme auquel je ne serai jamais prêt à renoncer, ce n'est pas seulement l'idée critique ou la posture questionnante [. . .] C'est plutôt une certaine affirmation émancipatoire et messianique, une certaine expérience de la promesse qu'on peut tenter de libérer de toute détermination métaphysico-religieuse, de tout messianisme. (Spectres, 147)

C'est au regard de cette « expérience de la promesse » que la vigilance mise en valeur par Derrida s'écarte d'une tradition qui serait seulement critique, hyper- ou auto-critique. Mais cela n'est pas moins vrai pour Marx puisque l'affirmation émancipatoire et messianique c'est justement l'esprit du marxisme que son héritier, la déconstruction, reçoit et réaffirme. Pourtant, personne ne confondrait jamais simplement l'attitude vigilante déconstructrice avec son antécédent marxiste. C'est dire que cette attitude a fondamentalement fait bouger quelque chose dans le regard vigilant, qu'elle l'a non seulement retourné contre lui-même, mais fait ressortir la façon dont il pivote afin de veiller sur ce qui peut venir depuis l'autre direction et la direction de l'autre.

Spectres de Marx prend son point de départ dans ce regard pivotant qui se tient en éveil devant l'esprit -le spectre ou le fantôme- qui revient, devant le revenant qui ouvre la pièce en se montrant encore une fois à ceux qui « watch the minutes of

\footnotetext{
4 Prolegomena to Any Future Metaphysics, Introduction.
} 
the night », qui veillent pendant la nuit. Hamlet met en scène tout de suite la vigilance devant ce que les vigiles à leurs postes ont déjà vu deux fois et pourtant ils seront encore surpris quand cela revient interrompre leur colloque. Je cite la première scène:

Marcellus: What, has this thing appeared again tonight?

Bernardo: I have seen nothing.

Marcellus: Horatio says 'tis but our fantasy,

And will not let belief take hold of him

Touching this dreaded sight twice seen of us.

Therefore I have entreated him along

With us to watch the minutes of this night...

Bernardo: Last night of all,

When yon same star that's westward from the pole

Had made his course t'illume that part of heaven

Where now it burns, Marcellus and myself,

The bell then beating one-

Enter the Ghost ...

Marcellus: Peace, break thee off. Look where it comes again.

Le fantôme entre en scène. La veille veille à -elle ne sait pas ce qui peut venir. Cette vigilance n'anticipe pas, elle ne s'attend pas à une arrivée qui mettra fin à son attente; et pourtant, elle veille à l'arrivée d'un autre pour l'interrompre. Il y a donc attention tendue à l'avance vers ce qui néanmoins va la surprendre, la surmonter et l'interrompre.

Derrida a souvent mis en valeur cet état de se rendre prêt à l'interruption -dans Béliers, par exemple, ce texte qui est, parmi tant de choses, une méditation sur l'interruption à la mémoire de Hans-Georg Gadamer. Après avoir cité un commentaire de ce dernier à propos d'une certaine indécision dans un poème de Celan, Derrida déclare :

Plus que l'indécision elle-même, j'admire le respect marqué par Gadamer à l'endroit d'une indécision. Celle-ci semble interrompre ou suspendre le déchiffrement de la lecture mais en vérité elle en assure l'avenir. L'indécision tient à jamais l'attention en haleine, c'est-à-dire en vie, éveillée, vigilante, prête à s'engager dans tout autre chemin, à laisser venir, tendant l'oreille, l'écoutant fidèlement, l'autre parole, suspendue au souffle de l'autre parole et de la parole de l'autre -là même où elle pourrait sembler encore inintelligible, inaudible, intraduisible. L'interruption est indécise, elle indécide. (Béliers, 37-38).

Plus qu'une description, ces lignes déclarent une admiration pour le fait que Gadamer soit prêt à laisser le poème interrompre et « indécider » sa lecture. Par là, 
elles déclarent aussi que c'est avec cette vigilance attentive, " prête à s'engager dans tout autre chemin ", que le lecteur Derrida lui-même, «tendant l'oreille, l'écoutant fidèlement », se tient prêt pour l'interruption. "Tendant l'oreille » : il s'agit donc, dans cette vigilance admirée, d'oreilles tournées ou tendues vers l'autre parole, qui est venue interrompre un déchiffrement trop certain du sens.

Je souligne le sens auditif parce que l'idée de vigilance ou de la veille pourraient autrement suggérer surtout l'attention tendue vers quelque chose de visible, comme dans l'exemple suprème de Hamlet où les gardes de nuit sont en garde pour « this dreaded sight twice seen of us », cette " redoutable vision deux fois vue par nous », le Spectre qui, du moins tant qu'il reste à leur portée, va et vient en silence. Pour Derrida, la vigilance n'est pas moins -c'est peut-être encore plus-ce qui peut s'entendre, être relevé par des oreilles tendues vers l'interruption qui vient de l'autre. Pourtant, étant donné que la vigilance dont il s'agit ici est aussi bien celle d'une lecture qui laisse son mouvement s'interrompre par l'autre parole, cette oreille écoutant fidèlement s'accorde à une virtualité intérieure : une voix, un bruit, ou bien, comme Derrida le soulignera dans un autre texte, un ton, c'est-à-dire, ce qui « ne se laisse pas reprendre par l'analyse -linguistique, sémantique ou rhétorique-d'une parole » (D'un ton apocalyptique, 93-94).

Mais qu'il soit configuré comme auditif ou visuel, Derrida prend en compte et fait appel à un espace de vigilance qui occupe ce qui se représente couramment comme l'intériorité d'un sujet. Cette représentation doit présupposer un rapport intrapsychique qui reste entre moi et moi-même, consciemment ou inconsciemment. Dès ses premiers écrits sur l'analyse husserlienne des signes, de la parole et de la langue, Derrida a fortement contesté cette présupposition de la présence immédiate et sans médiation d'un soi à soi-même dans la "vie solitaire de l'âme ».5 $\mathrm{Au}$ contraire, soutient Derrida, le rapport à soi n'est possible et ne peut s'intérioriser qu'à différer et espacer quelque non-soi, c'est-à dire, il ne peut que passer par ce que Derrida se mettra alors à appeler la différance. Il s'ensuit des conséquences fondamentales désormais pour toute tentative de représenter et de décrire, afin d'en rendre compte, l'expérience de l'intériorité comme espace d'un rapport à quelque non-soi, quelque altérité, quelqu'autre et, en fait, plus d'un.

Et ces autres en nous regardent, ils sont vigilants. À propos, par exemple, du roman d'Henri Thomas Le Parjure, dont le personnage principal semble avoir été calqué sur la jeunesse de son ami Paul de Man, Derrida écrit:

Car si tremblante et indécidable que restât, si suspendue que reste aujourd'hui encore la référence du roman et du « personnage » de fiction à notre ami de Man, nous ne pouvions pas ne pas être à la fois hantés par la mémoire que nous gardons de lui. Nous ne pouvions pas ne pas nous savoir en quelque sorte intérieurement observés par lui, par la

5 See Speech, esp. chaps. 3-5. 
vigilance spectrale de son regard, même si cette quasi-« présence » n'atténuait en rien notre liberté. Elle aiguisait même en vérité notre responsabilité. 6

Avec ce commentaire on pourrait se trouver de nouveau sur les remparts d'Elsinore où, selon Derrida, « [c] ette chose nous regarde cependant et nous voit ne pas la voir quand elle est là » (Spectres, 26). La vigilance spectrale intériorisée est asymétrique du moment que le regard de l'autre ne peut plus se croiser, le « ne ... plus » marquant la temporalité de l'interruption et du deuil. Elle nous rappelle que la veillée, comme le wake, nomme cette garde montée auprès des morts, auprès de tous ceux dont l'image et le regard nous observera désormais intérieurement tout en aiguisant notre responsabilité. 7

Soyez vigilants afin de tendre l'oreille vers la différence du ton apocalyptique qui exhorte: «Veillez : la fin s'approche ». D'un ton apocalyptique adopté naguère en philosophie évoque « le désir énigmatique de la vigilance, de la veille lucide, de l'élucidation, de la critique et de la vérité » qui se laisse appeler à la fois par ce discours apocalyptique promettant la révélation imminente et finale de la vérité, la vérité de la fin et comme la fin, et par tout discours qui, comme celui-ci de Derrida, voudrait « déconstruire le discours apocalyptique lui-même et avec lui tout ce qui spécule sur la vision, l'imminence de la fin, la théophanie, la parousie, le jugement dernier » (D'un ton apocalyptique, 64-65). La déconstruction du discours apocalyptique passe par l'attention vigilante à tout ce qui, rompant l'unité du ton ou de la voix, multiplie les routes pour l'envoi du sens.

Afin de désigner cette déroute générale de la destination et de la fin, Derrida propose le mot allemand Verstimmung -dérangement, désaccord- qui garde la trace de Stimme, la voix. Le ton apocalyptique ne serait, écrit-il, que

l'effet d'un déraillement généralisé, d'une Verstimmung multipliant les voix et faisant sauter les tons, ouvrant chaque parole à la hantise de l'autre dans une polytonalité immaîtrisable, avec greffes, intrusions, parasitages. La Verstimmung généralisée, c'est la possibilité pour un autre ton, ou le ton d'un autre de venir à n'importe quel moment

\footnotetext{
${ }^{6}$ Derrida, "Le parjure, peut-être (« brusques sautes de syntaxe »), Etudes françaises, 38, 1-2, p. 26.

7 On this interiorized gaze of the other, see Derrida, "The Deaths of Roland Barthes": "Roland Barthes looks at us (inside each of us, so that each of us can then say that Barthes's thought, memory, and friendship concern only us), and we do not do as we please with this look, even though each of us has it at his or her disposal, in his own way, according to her own place and history. It is within us but it is not ours; we do not have it available to us like a moment or part of our interiority" ("Barthes," 44), and "By Force of Mourning": "We are all looked at, I said, and each one singularly, by Louis Marin. He looks at us. In us" ("Mourning," 161); see also, infra, "To Follow", xx.
} 
interrompre une musique familière [. . . ] tout à coup, un ton venu d'on ne sait où coupe la parole, si on peut dire, à celui qui semblait tranquillement déterminer (bestimmen) la voix et assurer ainsi l'unité de destination, l'identité à soi de quelque destinataire ou destinateur. La Verstimmung, si l'on appelle ainsi désormais le déraillement, la saute du ton comme on dirait la saute d'humeur, c'est le désordre ou le délire de la destination (Bestimmung) mais aussi la possibilité de toute émission. L'unité du ton, s'il y en avait, serait certes l'assurance de la destination mais aussi la mort, une autre apocalypse.

(D'un ton apocalyptique, 67-68)

Ce passage peut rappeler cette indécision qui, comme nous avons lu il y a un instant, « tient à jamais l'attention en haleine, c'est-à-dire en vie, éveillée, vigilante, prête à s'engager dans tout autre chemin, à laisser venir, tendant l'oreille, l'écoutant fidèlement, l'autre parole». Soyez à l'écoute fidèle pour la Verstimmung de la Stimme, le délire de la destination dans le ton, la voix, le discours : voilà le mot d'ordre -en anglais je dirais plutôt le watchword- de la vigilance déconstructrice, de Derrida le veilleur.

Et cette vigilance est appelée à s'exercer dès la première phrase de ce texte : « Je parlerai donc d'un ton apocalyptique en philosophie ». Cette phrase, tout à fait régulière dans sa grammaire et sa syntaxe, ne se laisse pourtant pas traduire, du moins en anglais mais sans doute aussi dans d'autres langues, sans quelque supplément ou artifice pour marquer la division déroutante qui y a lieu entre plus d'une destination de sens. ${ }^{8}$ « Je parlerai d'un ton apocalyptique », c'est-à-dire, ce ton sera mon sujet, alors que la même phrase pourrait tout aussi bien annoncer le ton apocalyptique du discours qui va suivre. La phrase réussit ainsi une démonstration remarquablement économique de plusieurs choses à la fois. En situant le chevauchement du discours apocalyptique avec celui qui le déconstruirait, elle devient le support audible de cette hantise ou ce parasitage d'une parole par une autre qui divise toute la scène de la destination entre plus d'un destinateur, plus d'un destinataire, plus d'un sens. D'autant plus que cette hantise est réciproque : aucun des deux discours ne peut empêcher son interruption par l'autre, dans la condition d'une Verstimmung généralisée.

Il faut donc imaginer que quand elle fut prononcée pour la première fois, cette phrase initiale fut suivie d'une pause, même minime, pour laisser à ses premiers destinataires le temps de l'entendre se répéter en silence et pour donner place à une écoute fidèlement avec plus d'une oreille. ${ }^{9}$ Comme Derrida écrira un peu plus tard, à propos de Nietzsche: « tout revient à l'oreille avec laquelle vous pouvez m'entendre » (Otobiographies, 38).

\footnotetext{
8 John Leavey translates "I will speak then of/in an apocalyptic tone in philosophy" ("Tone," 117).

9 Both the original edition and the translation format this pause as a blank line after the initial sentence.
} 
Il est temps de conclure, mais je ne le ferai pas sans avoir avoué un doute à propos de cette écriture du wake vigilant que je poursuis : il est possible et même probable qu'elle travaille à désavouer ce qui ressemble plutôt à une écriture du rêve. Si je reprenais le récit de l'oubli du mot wake, peut-être devrais-je recommencer à partir, par exemple, du dernier paragraphe de La Grammatologie, où on peut lire ceci: «L'opposition du rêve à la vigilance, n'est-ce pas aussi une représentation de la métaphysique ? Et que doit être le rêve, que doit être l'écriture si, comme nous le savons maintenant, on peut rêver en écrivant ? Et si la scène du rêve est toujours une scène de l'écriture » (Grammatologie, 444-45). Plus de trente ans après les avoir posées, ces questions vont résonner encore pour Derrida en tant qu'il voudrait penser -ou rêver- la " possibilité de l'impossible ». Tout comme Adorno, il sait qu'une telle pensée ne peut pas opposer sa vigilance, son wakefulness, aux rêves ; au contraire, il faut veiller sur les rêves afin de les bannir sans les trahir. C'est cette réflexion de Walter Benjamin que Derrida reprend à sa manière dans Fichus:

Bannir le rêve sans le trahir [. . .]: se réveiller, cultiver la veille et la vigilance, tout en restant attentif au sens, fidèle aux enseignements et à la lucidité d'un rêve, soucieux de ce que le rêve donne à penser, surtout quand il nous donne à penser la possibilité de l'impossible. La possibilité de l'impossible ne peut être que rêvée, mais la pensée, une tout autre pensée du rapport entre le possible et l'impossible, cette autre pensée [. . . a a peut-être plus d'affinité que la philosophie même avec le rêve. Il faudrait, tout en se réveillant, continuer de veiller sur le rêve. (Fichus, 20).

Il faudrait, tout en se réveillant, continuer de veiller sur le rêve: afin de veiller sur l'avenir, s'attendre à l'inattendu, tendre l'oreille pour le changement de ton, se tenir prêt à l'interruption. 